\title{
The Effect of Self-care Education on knowledge and Attitude in Patients with Asthma: A Randomized Clinical Trial
}

\author{
Amir Sadghi ${ }^{1}$, Somyhe Kokabi ${ }^{2}$, Khodayar Oshvandi ${ }^{3}$, Lilly Tapak ${ }^{4}$ \\ 1. Department of Pediatric Nursing and Mental Health, Faculty of Nursing \& Midwifery, Hamadan University of Medical \\ Sciences, Hamadan, Iran \\ 2. MSc, Faculty of Nursing \& Midwifery, Hamadan University of Medical Sciences, Hamadan, Iran \\ 3. Maternal and Child Care Research Center, Faculty of Nursing \& Midwifery, Hamadan University of Medical Sciences, \\ Hamadan, Iran \\ 4. Department of Biostatistics, School of Health, Hamadan University of Medical Sciences, Hamadan, Iran
}

\begin{tabular}{|c|c|}
\hline Article Info & ABSTRACT \\
\hline $\begin{array}{l}\text { Received: 2019/02/03; } \\
\text { Accepted: 2019/08/17; } \\
\text { Published Online: 2019/08/28 }\end{array}$ & $\begin{array}{l}\text { Introduction: Asthma is one of the most common chronic respiratory diseases and } \\
\text { one of the major problems in health systems in many societies around the world } \\
\text { including Iran. Self-care is one of the most important factors in raising the awareness } \\
\text { and attitude of individuals. The purpose of this study was to investigate the effect of } \\
\text { self-care education on knowledge and attitude of patients with asthma. }\end{array}$ \\
\hline$\underline{10.30699 / \mathrm{sjhnmf} .27 .5 .306}$ & Methods: In this quasi-experimental study, 104 patients with asthma were selected \\
\hline Original Article & $\begin{array}{l}\text { control }(n=52) \text {. Both groups filled the questionnaire of awareness and attitude before } \\
\text { the intervention. The self-care group was trained in four sessions of } 60 \text { minutes. The } \\
\text { questionnaire was filled again this time by both groups, } 8 \text { weeks after the intervention. } \\
\text { and the data was analyzed using SPSS } 16 \text {, descriptive statistics, independent t-test and } \\
\text { paired t-test at } 95 \% \text { confidence interval. }\end{array}$ \\
\hline
\end{tabular}
and read the article online

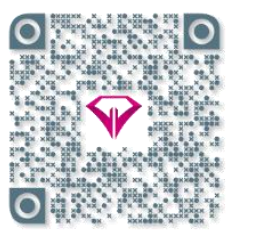

Results: The results showed that there was no significant difference between the mean and standard deviation of knowledge and attitude scores before and after selfcare education $(P>0.05)$. There was a statistically significant difference between the mean and standard deviation of the knowledge and attitude of the experimental and control groups after the training $(P<0.05)$. The results showed that the knowledge and attitude of the experimental group significantly improved after the intervention.

Conclusion: The results of this study showed that self-care education is effective on knowledge and attitude in asthmatic patients. Therefore, considering the high prevalence of this disease and its economic and social burden, self-care education is recommended to improve the knowledge and attitude of asthmatic patients.

Keywords: Self-Care, knowledge, Attitude, Asthma

Corresponding Information: Somyhe Kokabi, Faculty of Nursing \& Midwifery, Hamadan University of Medical Sciences, Hamadan, Iran. E-mail: kokabiam.s@gmail.com

Copyright ( $\odot$ 2019, This is an original open-access article distributed under the terms of the Creative Commons Attribution-noncommercial 4.0 International License which permits copy and redistribution of the material just in noncommercial usages with proper citation.

\section{How to Cite This Article:}

Sadeghi A, Kokabi S, oshvandi K, Tapak L. The Effect of Self-care Education on knowledge and Attitude in Patients with Asthma: A Randomized Clinical Trial. Avicenna J Nurs Midwifery care. $2019 ; 27$ (5) :306-314 


\section{تأثير آموزش خودمراقبتى بر آكاهى و نكَرش بيماران مبتلا به آسم: يكى مطالعه كار آزمايى بالينى تصادفى}

$$
\text { امير صادقى'، سميه كوكبى'، خدايار عشوندى"، ليلى تاياك؟ }
$$

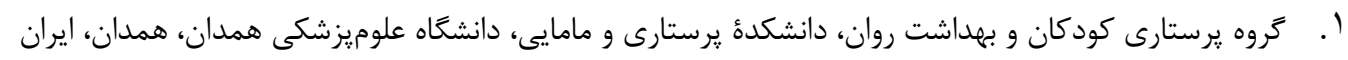

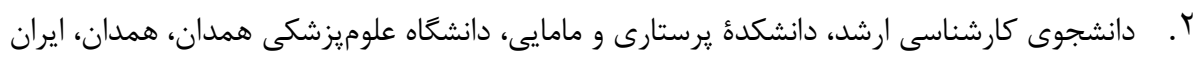

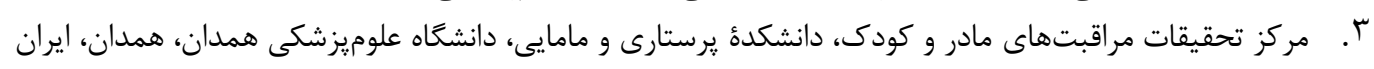

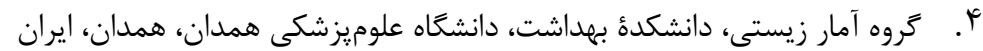

\begin{tabular}{|c|c|}
\hline جكيده & اطلاعات مقاله \\
\hline 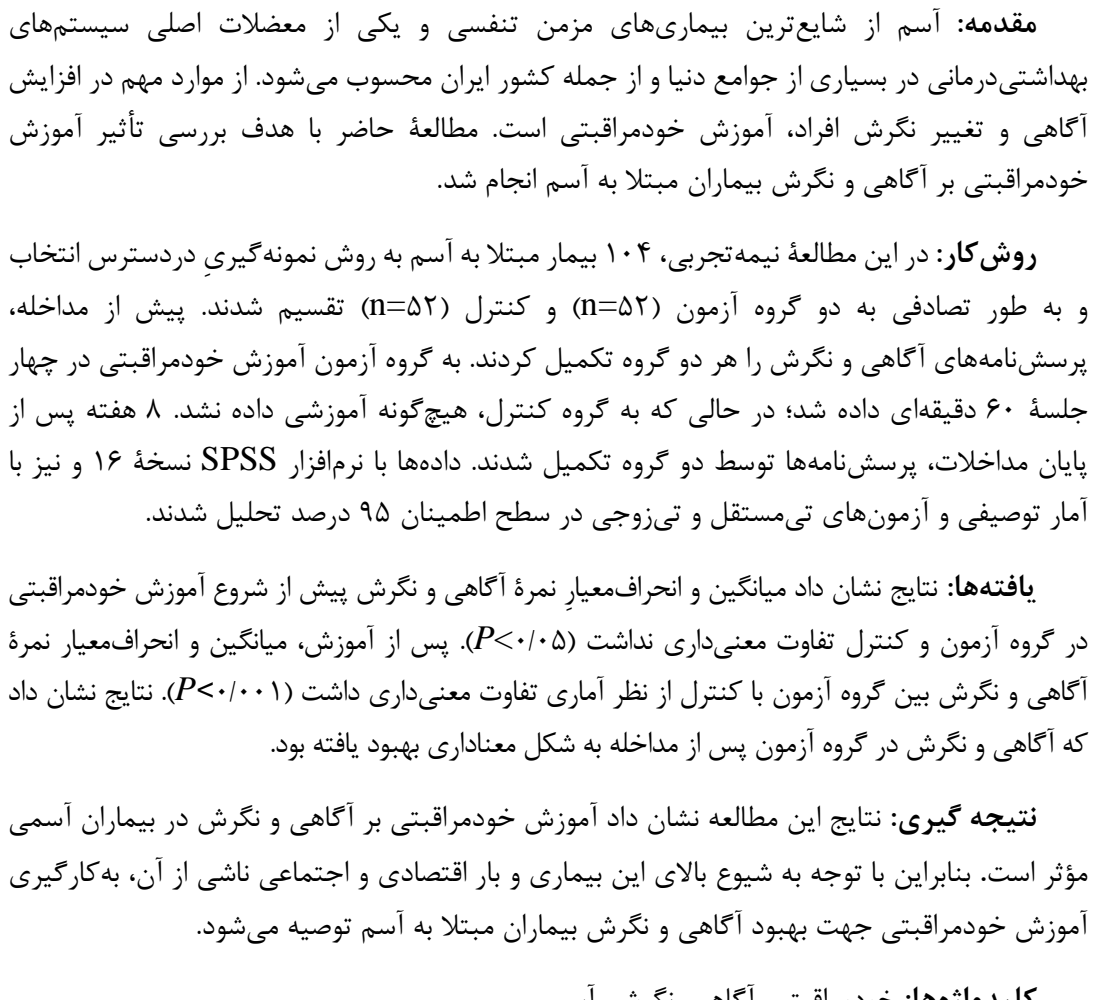 & 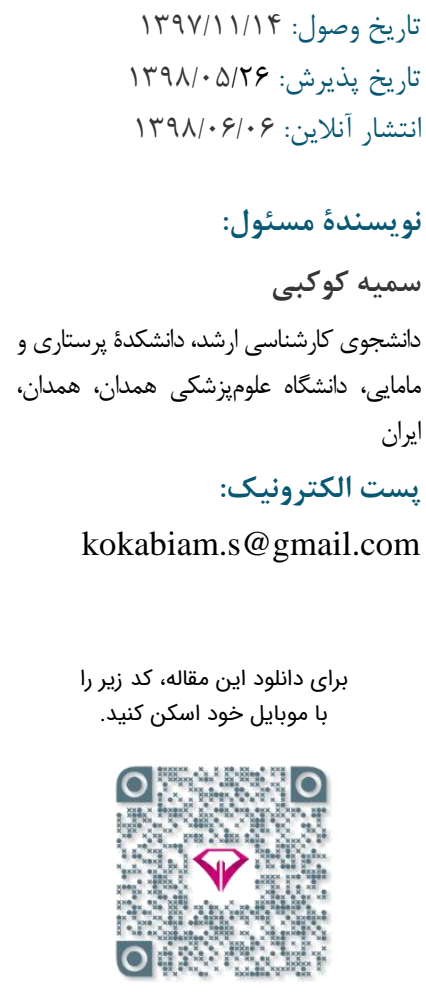 \\
\hline
\end{tabular}

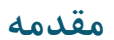

بهداشتى است و سالانه تعداد زيادى از مبتلايان به اين بيمارى

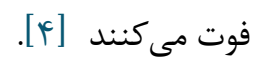

آسم بيمارى التهابى مزمن راههاى هوايى است كه موجب

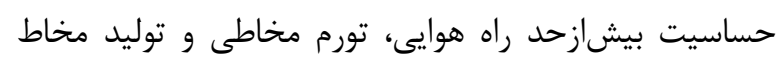

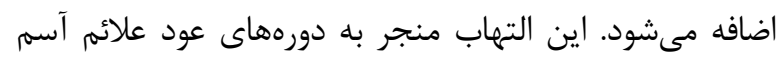

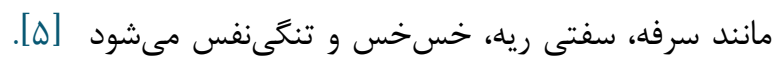

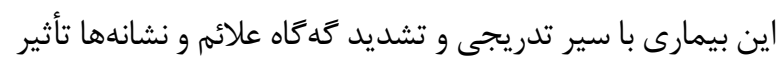

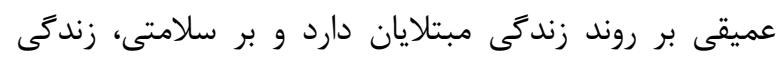

بيش از ه/ اميليارد نفر در سراسر دنيا به بيمارىهاى مزمن

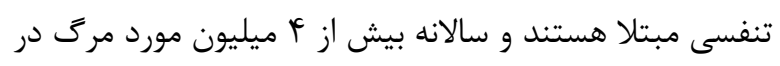

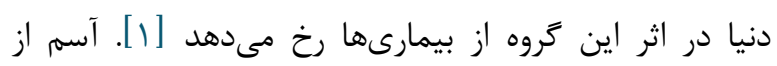

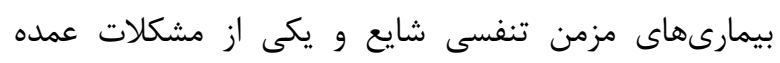

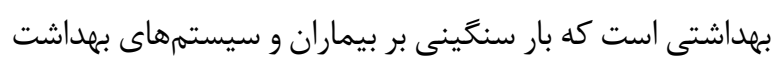

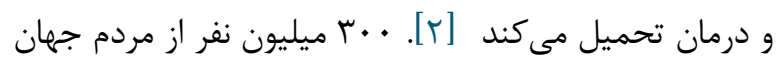

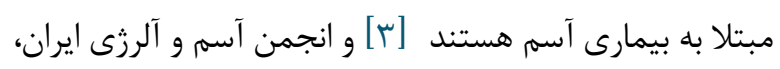

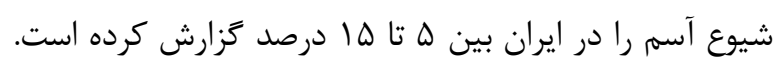

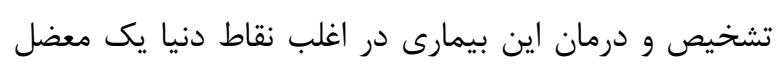


^• ץ تأثيرآموزش خودمراقبتى بر آكاهى و نكَرش بيماران مبتلا به آسم

بخشيد يا تغيير داد. آكاه نمودن و كسترش اطلاعات لازم درخصوص ارتقاى سلامت، باورهاى نادرست را از بين مىبرد و

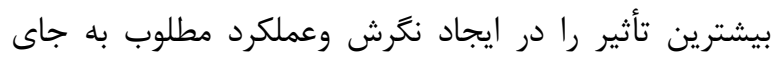

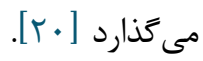

Zografos

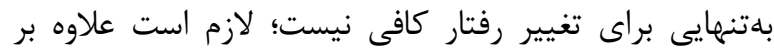

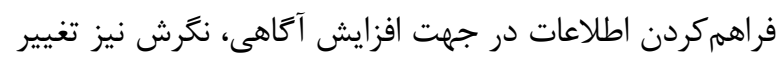

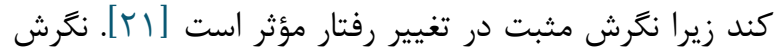
افراد به يديدهها مىتواند بر بسيارى از رفتارها تأثير بخذارد

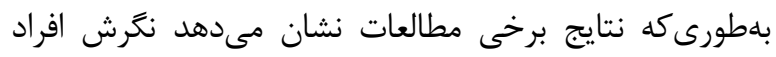

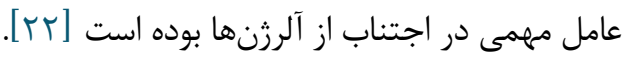

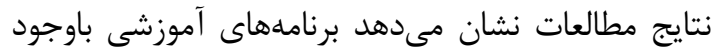
تنوع زيادى كه در شكل، محيط، گروه هدف، اهداف، شيوه

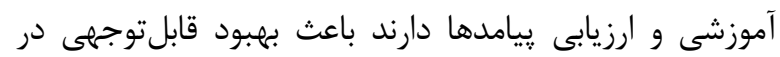

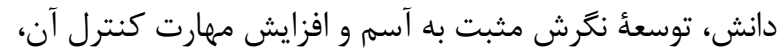
كاهش غيبت از مدرسه، افزايش فعاليت جسمى، افزايش ندان

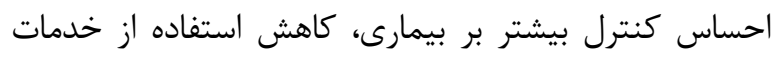
مراقبت بهداشتى مىشود [سب]. اكر جه برخى مطالعات بيانكر تأثير مثبت آموزش بر آثرا. آكاهى

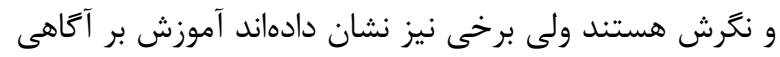

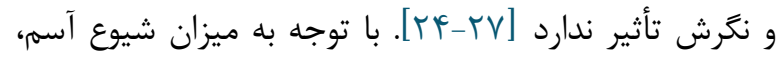

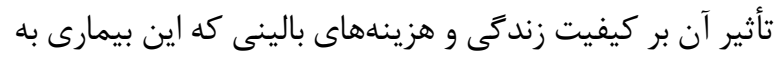
فرد و جامعه تحميل مى كند و نتايج متفاوتى كه در مطالعات مشابه مشاهده شده است و نيز وجود تحقيقات معدود در زمينه

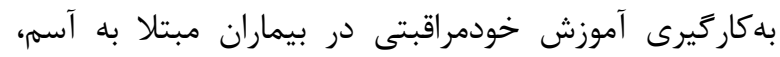

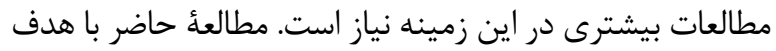

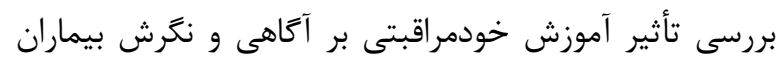

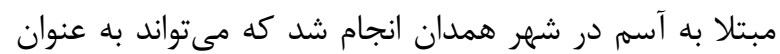

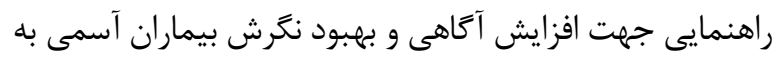

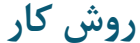

يزوهش حاضر يك كارآزمايى بالينى تصادفىشده است.

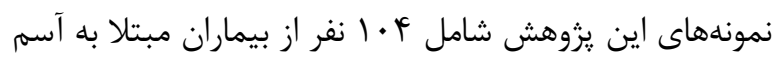
مراجعه كننده به كلينيك فوقتخصصى ريه در بيمارستان شهيد نديد

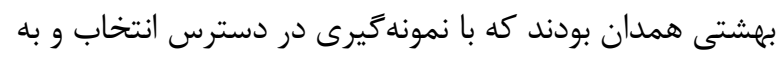
طور تصادفى (بلوكهاى أتايى) به دو كروه آزمون و كنترل (كروههاى Tله نفرى) تقسيم شدند.
خانوادَى، فعاليت گَروهى، اجتماعى و عملكرد شغلى آنها تأثير مى كذارد [عان.

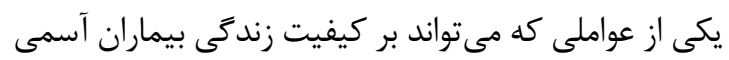

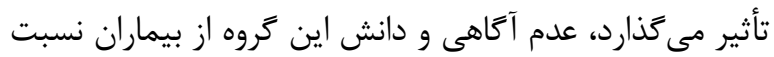

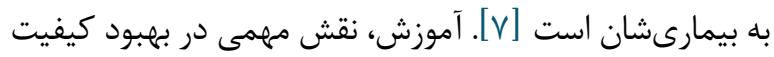

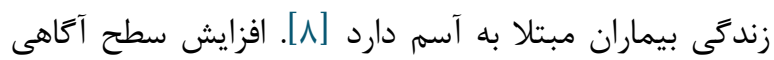

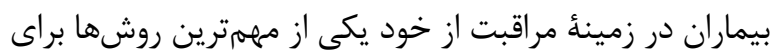
كنترل اين بيمارى است. با آموزش خودمراقبتى به جاى جاى تكيه

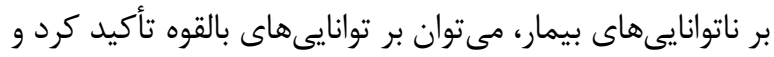

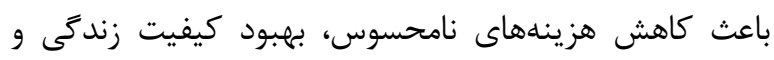
ارتقاء سطح فعاليتهاى روزمره شد [9]. خودمراقبتى به معنى فرايند حفظ سلامتى از طريق

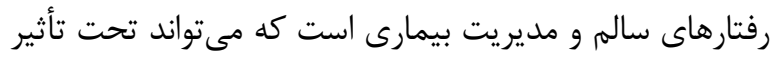

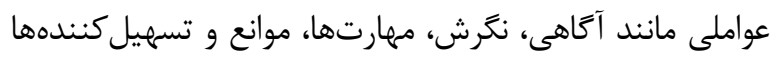

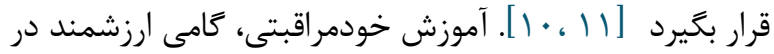
افزايش آكاهى و كمك به بيماران در كسب استقلال فردى و

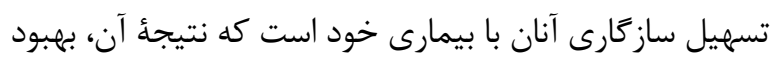
سلامت عمومى بيمار، شركت فعالانه در روند مراقبت از خود و

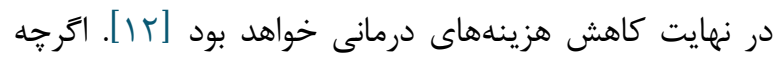

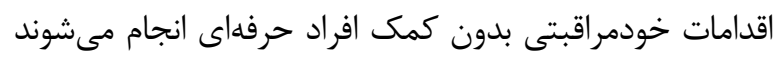

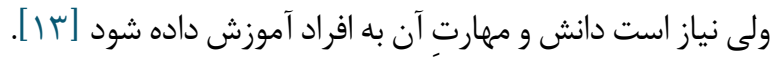
آموزش به بيمار به عنوان وظيفهاى مههم و يكى از استانداردهاى

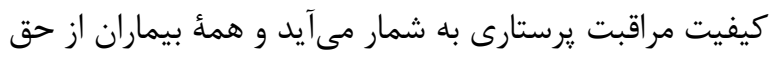

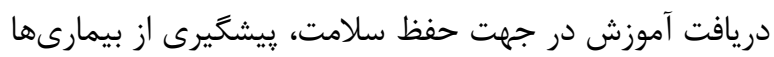

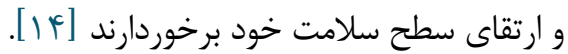
مطالعات انجامشده حاكى از كمبود آكاهى، نكَرش و عملكرد مناسب بيماران در مواجهه با بيمارىها و درنتيجه، رعايتنكردنٍ

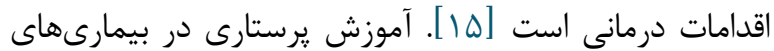
مختلف باعث افزايش آكاهى، تغييرات رفتارى در جهت بهات بهبودا،

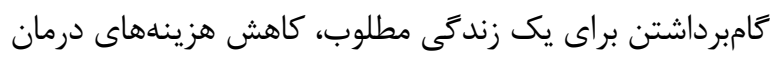

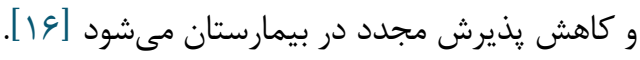

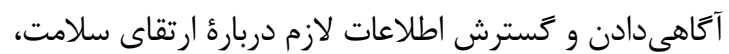

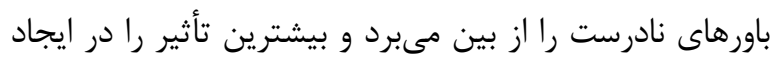

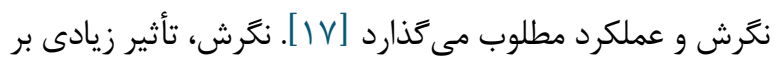
رفتار دارد و اكر فرد، نكرش منفى به موضوعى خاص داشته باشد نبايد انتظار داشت رفتارهاى مثبت و حمايتى از خود بروز دهد دارد

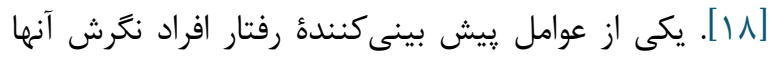

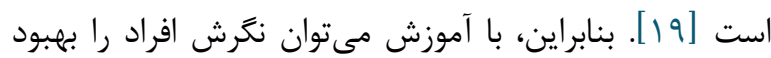




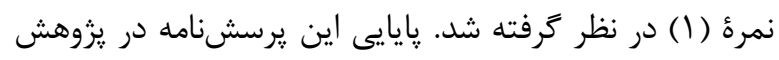

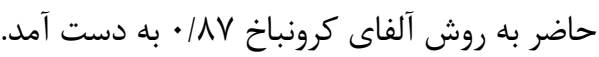

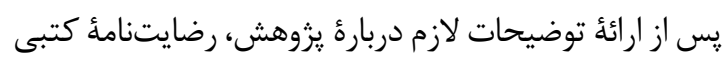

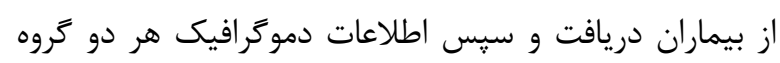

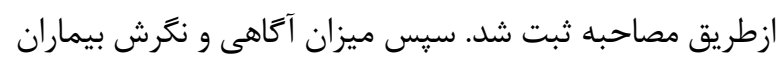

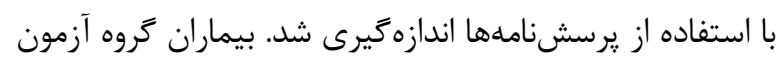

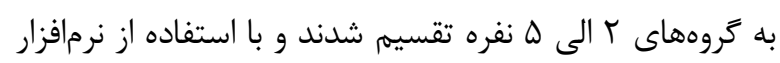

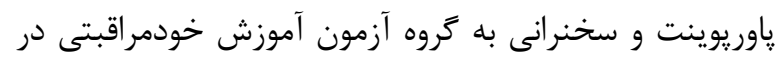

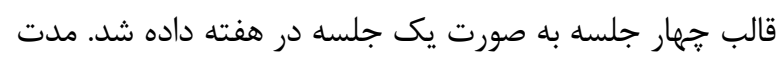

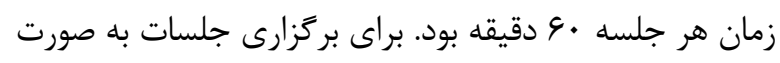

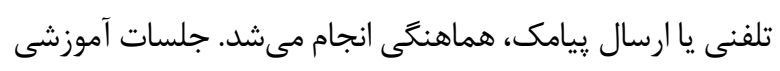

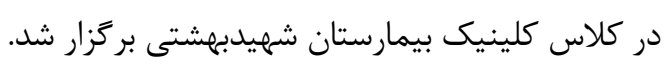

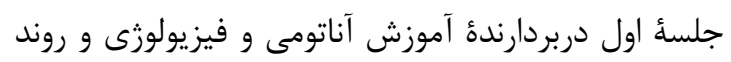

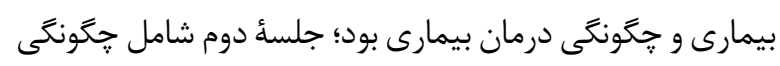

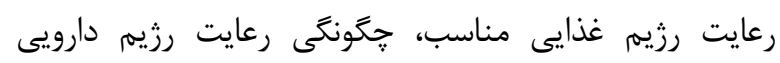

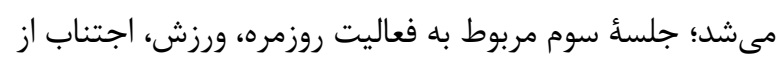

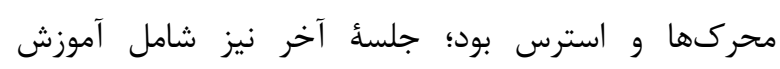

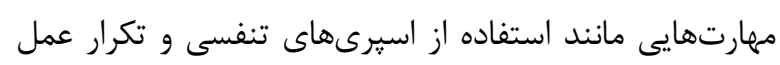

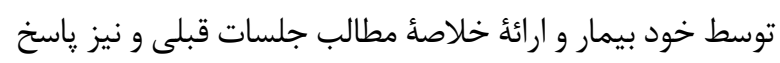

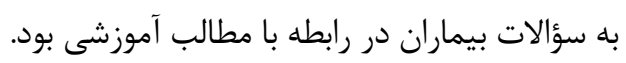

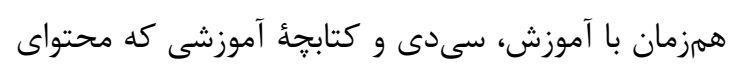

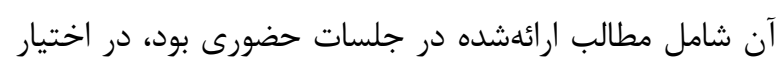

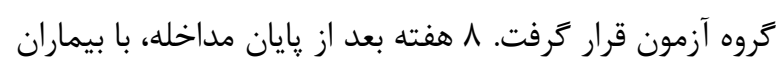

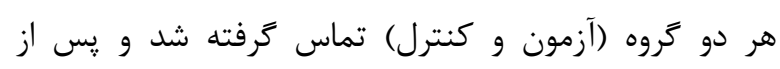

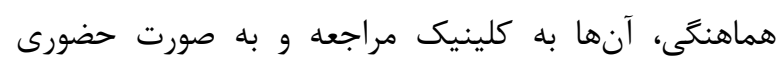

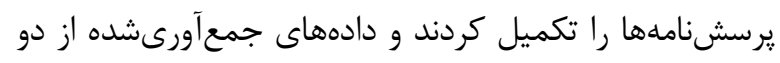

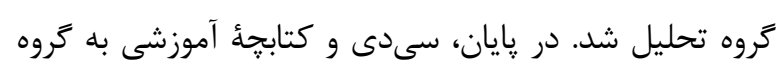

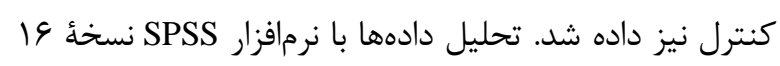

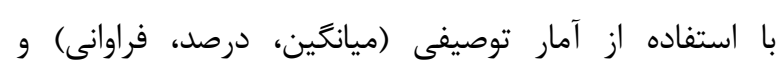

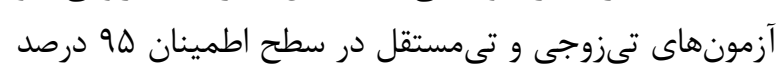

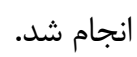

\section{يافته ها}

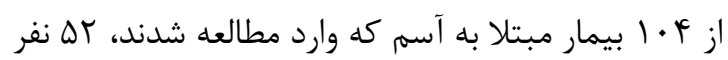

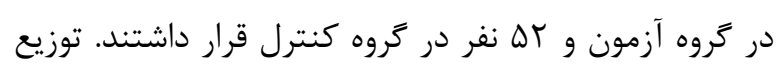

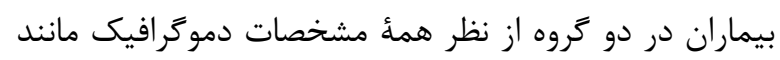

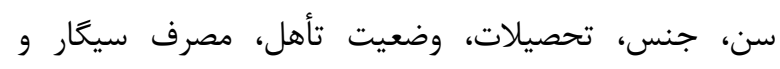

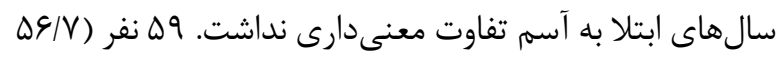

معيارهاى ورود به مطالعه عبارت بودند از ابتلاى فرد به

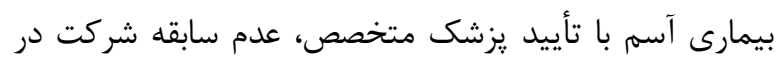

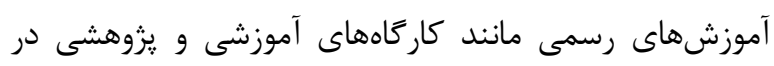

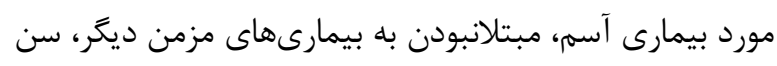

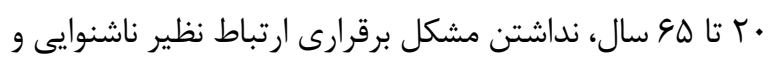

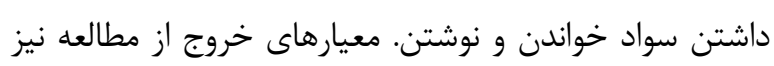

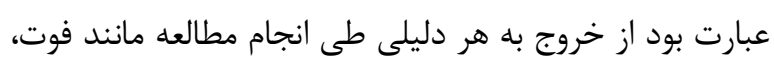

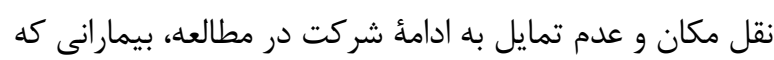

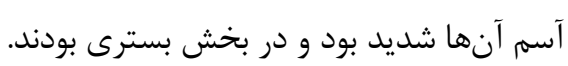

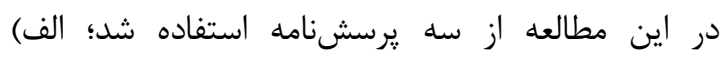

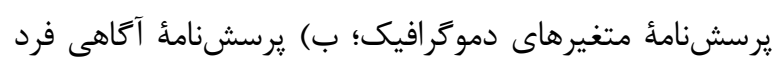

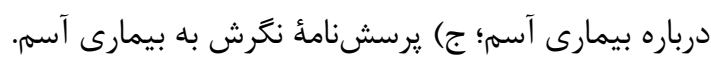

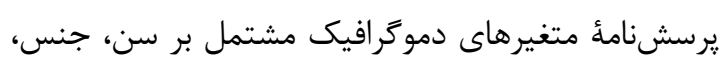
شغل، سطح تحصيلات، وضعيت تأهل، سالهاى ابتلا به آسم و

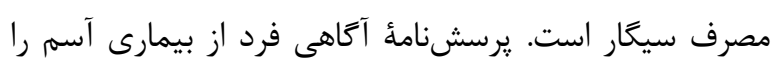

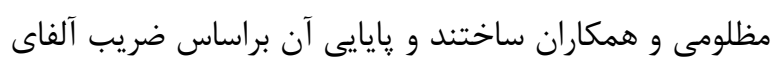

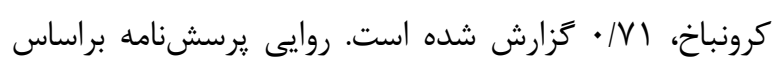

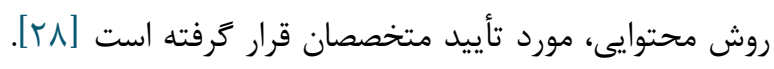

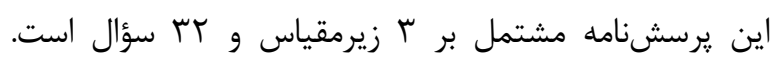

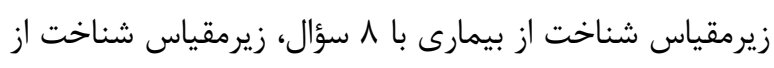

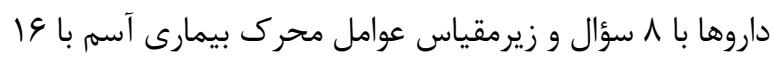

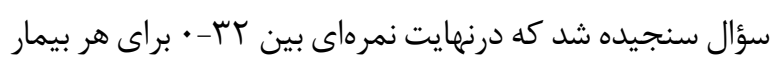

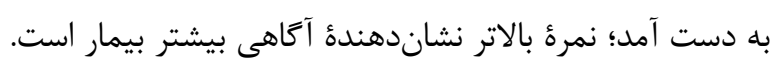

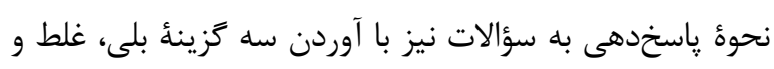

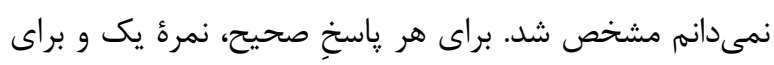

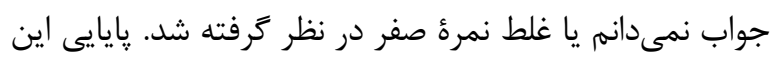

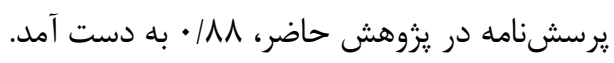

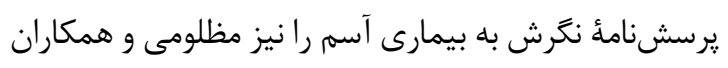

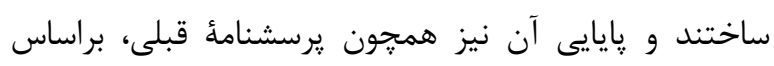

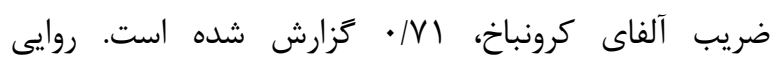

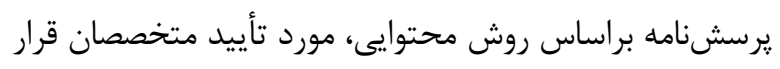

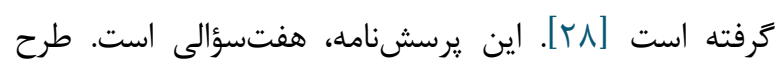

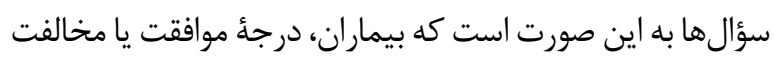

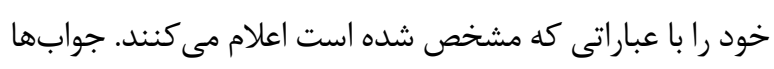

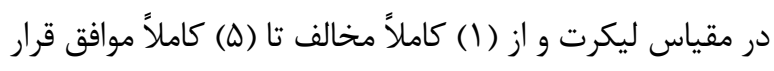

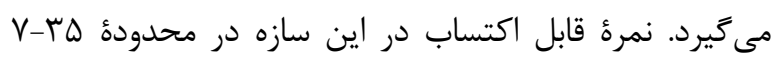

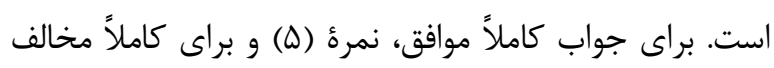


• اب تأثيرآموزش خودمراقبتى بر آكاهى و نكَرش بيماران مبتلا به آسم

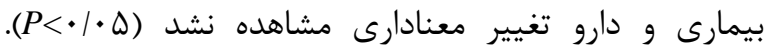

$$
\begin{aligned}
& \text { درصد) از آنان زن و لهF نفر (س/ آع درصد) مرد بودند. دادهها }
\end{aligned}
$$

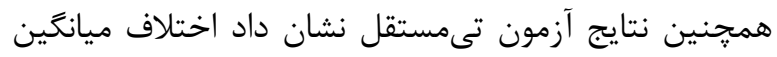

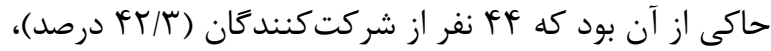

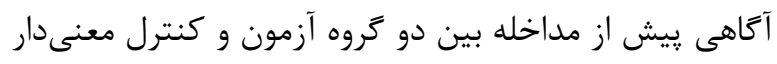

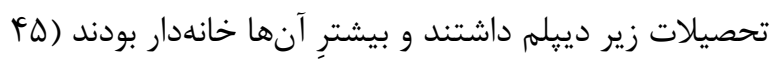

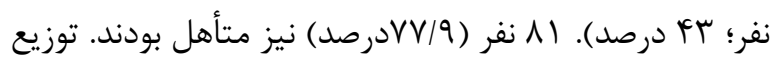

$$
\begin{aligned}
& \text { بيماران در دو گروه ار نظر مشخصات دموكرافيگ تفاوت } \\
& \text { ميانكين آكاهى ڤس از مداخله بين دو گروه آزمون و كنترل }
\end{aligned}
$$

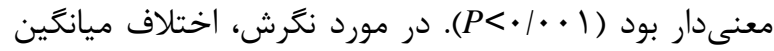

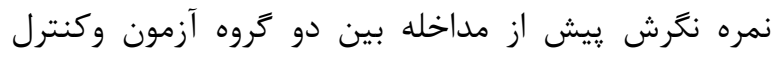

$$
\begin{aligned}
& \text { معنى دار نبود (99/9= •P). درحالى كه همين آزمون نشان داد }
\end{aligned}
$$

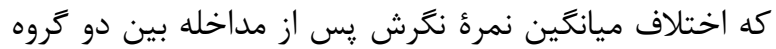

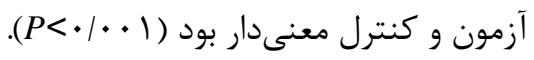

$$
\begin{aligned}
& \text { معنى دارى نداشت. }
\end{aligned}
$$

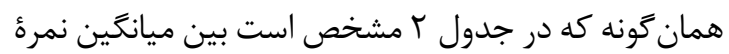

$$
\begin{aligned}
& \text { آكاهى و ابعاد بيماران در گروه آزمون بيش و يس إن از مداخله، }
\end{aligned}
$$

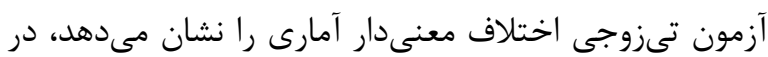

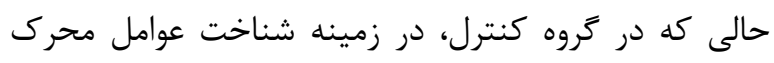

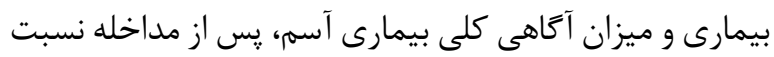

$$
\begin{aligned}
& \text { به بِيش از مداخله تغيير معنى دار وجود دارد، اما در شناخت }
\end{aligned}
$$

جدول ا. مقايسه اطلاعات دموكرافيك بين دو كروه كنترل و آزمون ييش از مداخله

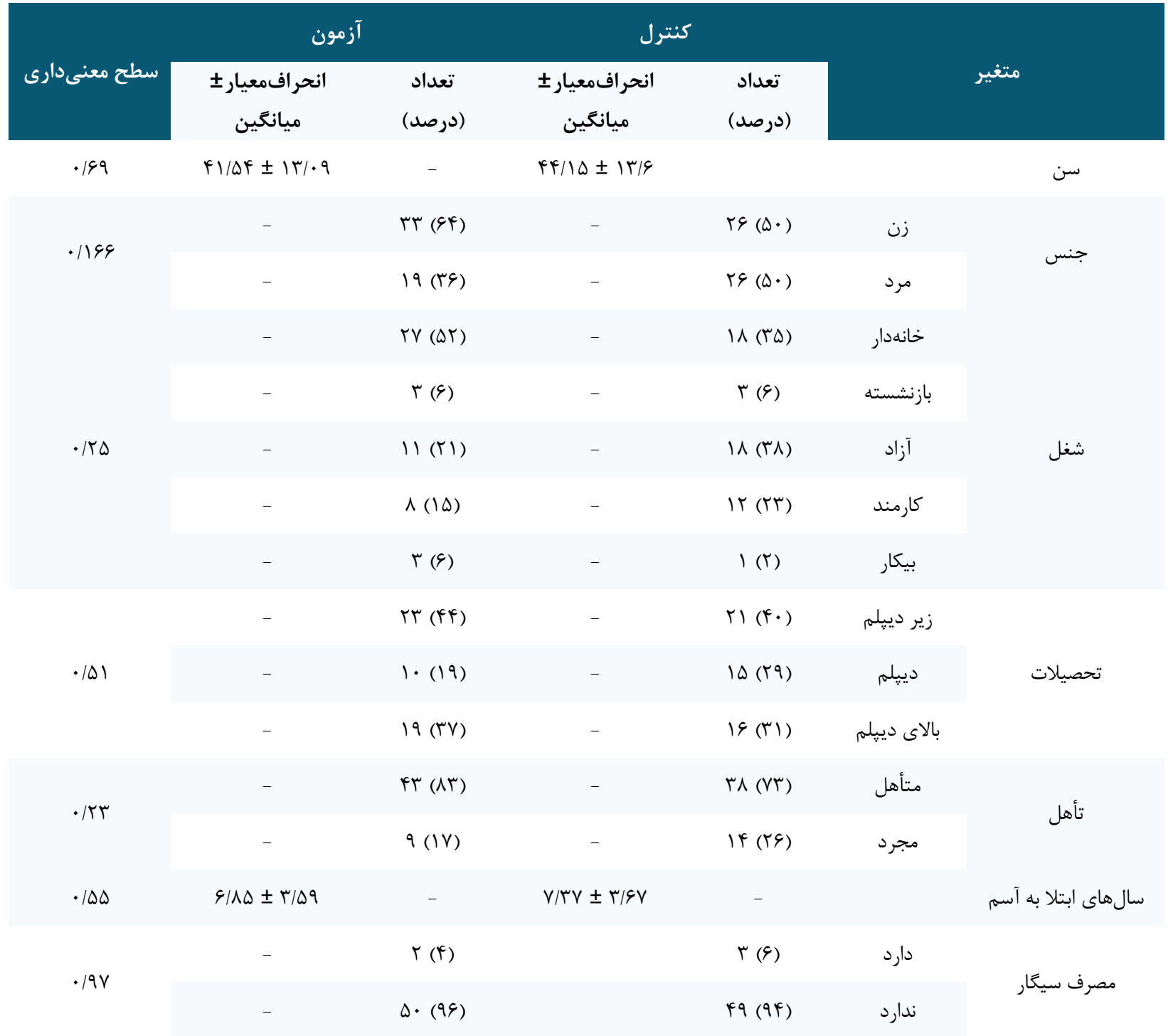


جدول r. مقايسهُ ميانگين آتاهى و ابعاد آكَاهى و نغَرش بين دو تروه آزمون و كنترل بيش و يس از مداخله

\begin{tabular}{|c|c|c|c|}
\hline نتيجه آزمون & يس از مداخله & 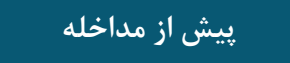 & \multirow[b]{2}{*}{ 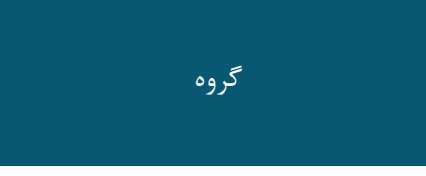 } \\
\hline $\begin{array}{c}\text { تى زوج } \\
\text { P-value }\end{array}$ & ميانغين 土 انحرافمعيار & ميانَين \ انحرافمعيار & \\
\hline$\cdot|\cdot Y|$ & $19 / 1 \pm r / 9$ & $\mid F / \Lambda V \pm F / q r$ & 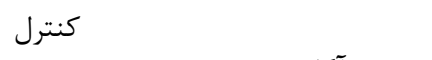 \\
\hline \multirow[t]{2}{*}{$\cdot 1 \cdot \cdots 1$} & $T \& / F T \pm T / T$ & $\mid F / \wedge \Delta \pm F / 9 \Delta$ & 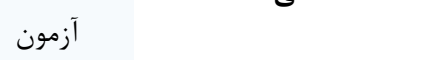 \\
\hline & $\cdot 1 \cdots \cdot 1$ & $\cdot 191$ & نتيجه آزمون تىمستقل P-value \\
\hline$\cdot / \cdots r$ & $|r /| \pm T / F V$ & $11 / r 1 \pm r / 9 r$ & \multirow{2}{*}{ ل ناخت عوامل محرى } \\
\hline \multirow[t]{2}{*}{$\cdot 1 \cdots \cdot 1$} & $\mid F / \Delta T \pm 1 / F r$ & $11 / 1 r \pm r / \Delta \mid$ & \\
\hline & $\cdot 1 \cdot \cdots 1$ & $\cdot / V T$ & نتيجه آزمون تىمستقل P-value \\
\hline$\cdot 114$ & $T / K r \pm 1 / \Delta T$ & $r \pm 1 / \Delta \varphi$ & \multirow{2}{*}{ 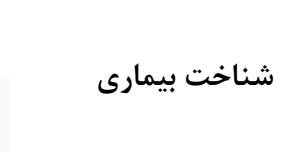 } \\
\hline \multirow[t]{2}{*}{$\cdot / \cdots 1$} & $9 / 19 \pm \cdot / 97$ & $r \pm 1 / \Delta r$ & \\
\hline & $\cdot 1 \cdot \cdots 1$ & 1 & نتيجه آزمون تىمستقل P-value \\
\hline$\cdot \mid \Delta F$ & $1 / 9 V \pm 1 / A r$ & $1 / \Delta \varphi \pm 1 / V \Lambda$ & \multirow{2}{*}{ شناخت دارو } \\
\hline \multirow[t]{2}{*}{$\cdot 1 \cdot \cdots 1$} & $\Delta / V I \pm 1 / Y Y$ & $1 / V r \pm 1 / \Lambda \Lambda$ & \\
\hline & $\cdot 1 \cdot \cdots 1$ & $\cdot \mid q \mu$ & نتيجه آزمون تىمستقل P-value \\
\hline$\cdot \mid \cdots \cdot$ & $r F / V q \pm F / V$ & $r F / \Lambda) \pm r / q r$ & \multirow{2}{*}{ 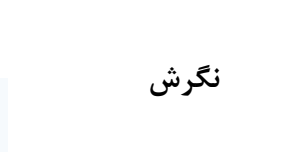 } \\
\hline .199 & $r / / r V \pm r / \Delta \varphi$ & $r Y / A V \pm \varphi$ & \\
\hline
\end{tabular}

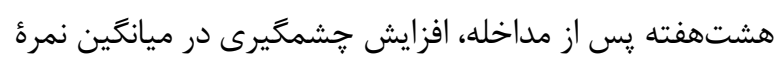

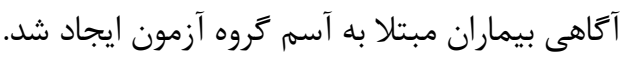

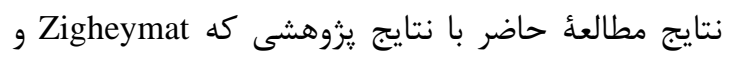

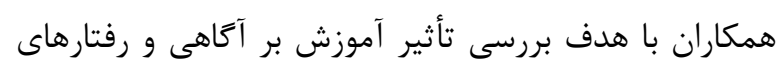

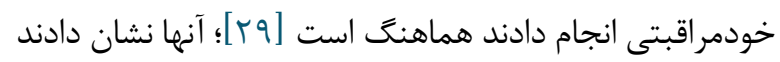

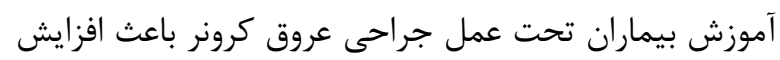

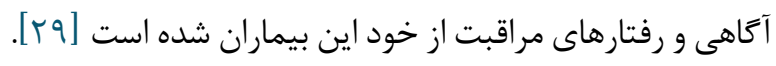

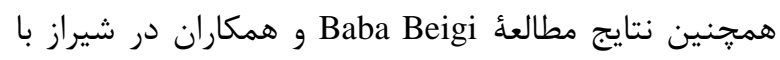

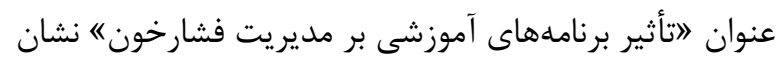

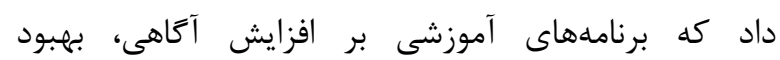

\section{بحث}

نتايج يزوهش حاضر نشان داد ميانگين نمره آكاهى و ابعاد

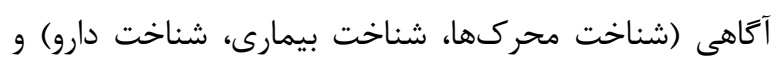

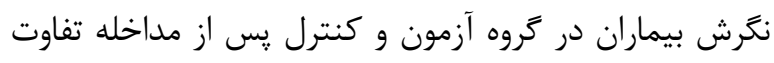

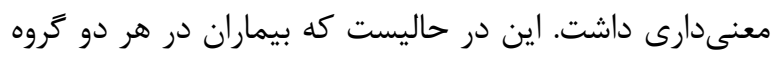

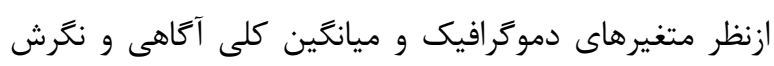

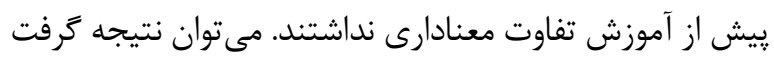

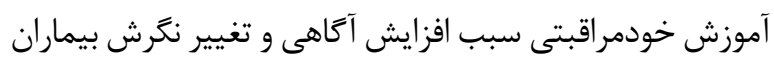

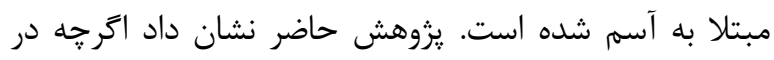

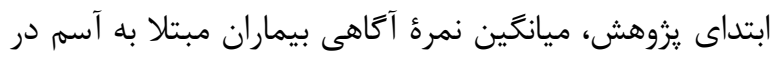

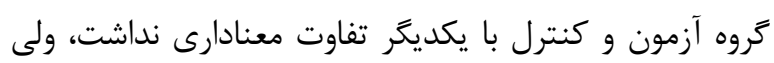


اين افزايش معنادار نبود كه علت آن احتمالاً مطالعه منابع مختلف توسط بيماران است. با استناد به يافتههاى مطالعه حاضر، افزايش دانش، درك و كاربرد دانش توسط بيماران با بان بان

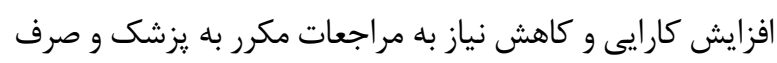
هزينهاى كزاف و كنار كذاشتنِ ادامةٔ درمان خواهد كاست.

\section{نتيجهُ نهايى}

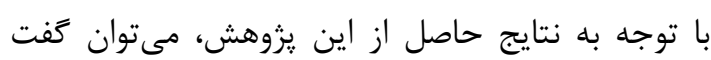

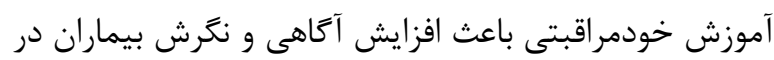

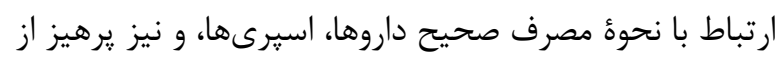

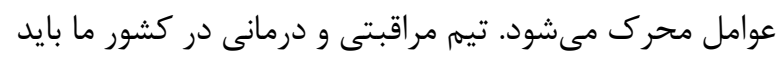

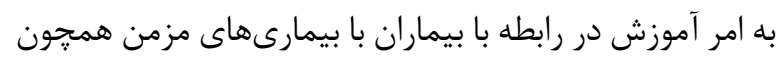
بيماران مبتلا به آسم، بيشتر توجه كنند.

\section{سياسگَزارى}

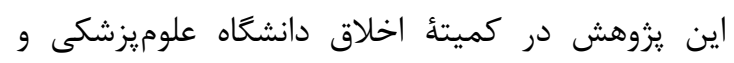

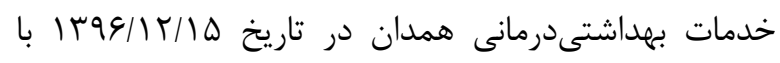

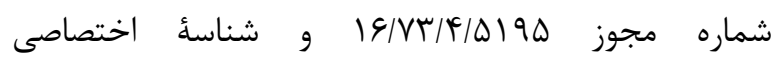
IR.UMSHA.REC.1396.725 در مركز كارآزمايى بالينى ايران IRCT2016.110025929N

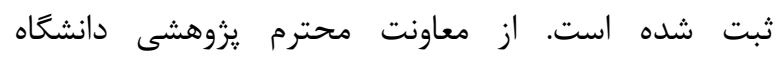

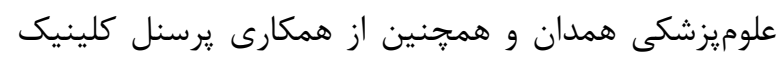

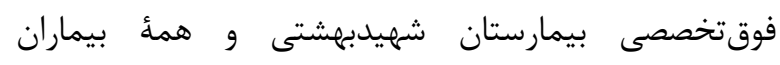

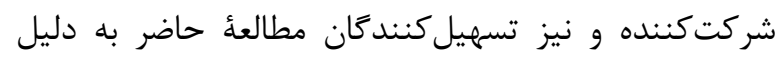

$$
\text { همكارى صميمانهشان قدردانى مى كنيم. }
$$

\section{تعارض در منافع}

بين نويسندكان هيجَّونه تعارضى در منافع وجود ندارد

$$
\text { منابع مالى }
$$

منابع مالى اين مطالعه توسط نويسندًان تامين شده است.
خودمراقبتى و كنترل عادات مضر در بيماران مبتلا به فشار

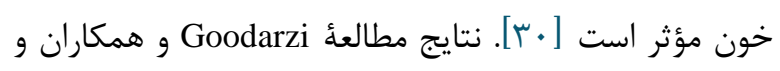

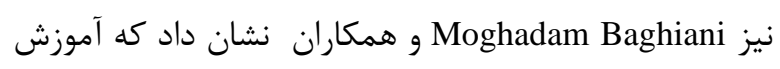

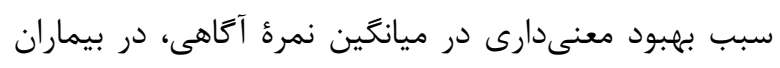

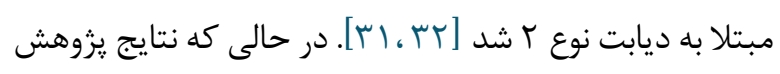
Bidi

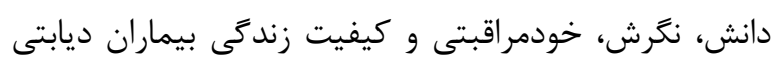

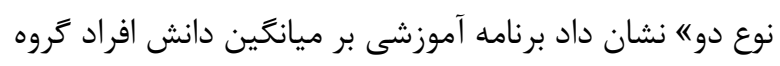

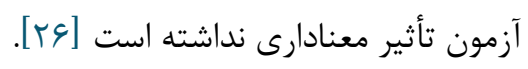

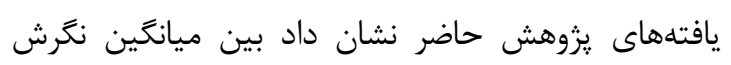

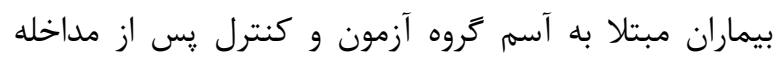

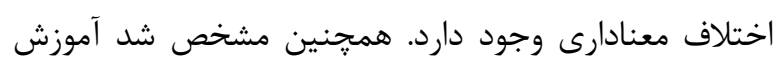

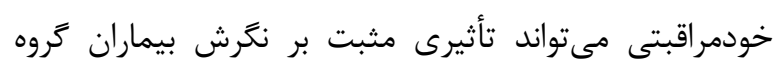

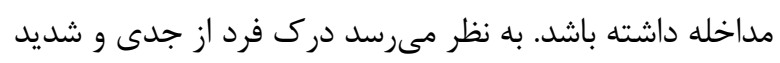

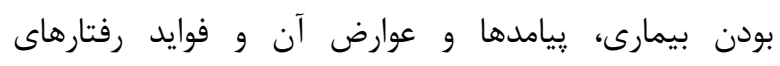

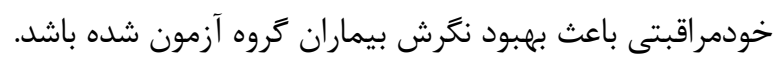

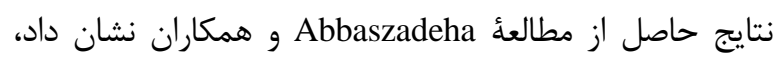
آموزش به بيماران مبتلا به انفاركتوس ميوكارد، اثرات مفيد واندان

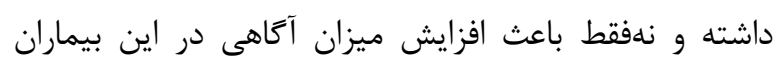

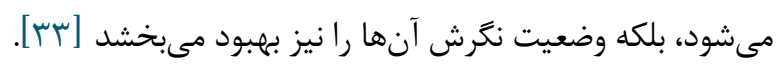

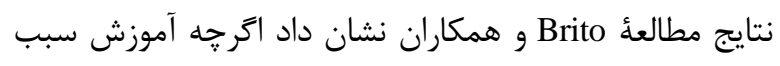

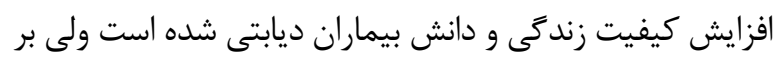

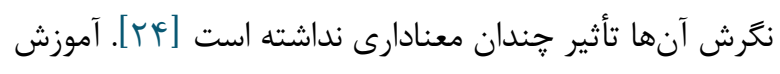

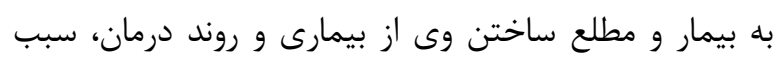

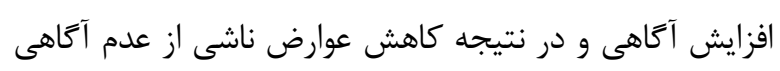

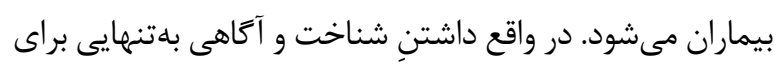

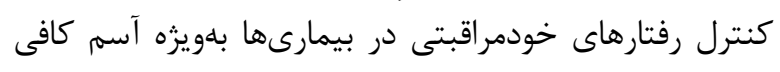

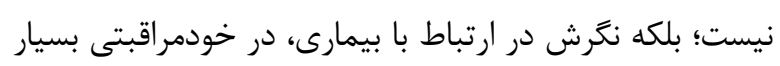

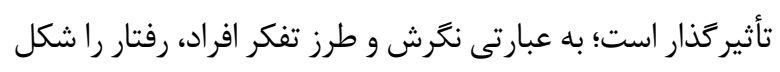

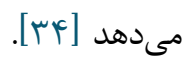

در اين يزوهش در ميانكَين نمره زيرمقياس شناخت

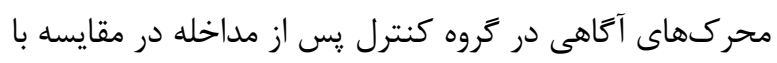
بيش از مداخله افزايش داشت ولى در مقايسه با كروه كره آزمون، 


\section{References}

1. Becker AB, Abrams EM. Asthma guidelines: the Global Initiative for Asthma in relation to national guidelines. Current Opinion in Allergy and Clinical Immunology. 2017; [DOI:10.1097/ACI.0000000000000346] [PMID]

2. Luyster FS, Teodorescu M, Bleecker E, Busse W, Calhoun W, Castro M, et al. Sleep quality and asthma control and quality of life in non-severe and severe asthma. Sleep and Breathing. 2012; 16(4):1129-37. [DOI:10.1007/s11325-011-0616-8] [PMID] [PMCID]

3. Uchmanowicz B, Panaszek B, Uchmanowicz I, Rosinczuk J. Clinical factors affecting quality of life of patients with Asthma. Patient Preference and Adherence. 2016; 10:579-89. [DOI:10.2147/PPA.S103043] [PMID] [PMCID]

4. Mohajjel Aghdam A, Hasankhani H, Gharemohammadlu R, Esmaeily M. Relation of patients self-efficacy with control-4 of asthma symptoms. Journal of Gorgan University of Medical Sciences. 2013; 15(2):70-6.

5. Oni O, Efosa Erhabor G, Oluboyo P. Does Health Related Quality of life in asthma patients correlate with the clinical indices? Journal of South African Family Practice. 2014; 56(2):134-8. [DOI:10.1080/20786204.2014.10855351]

6. Naveed S, Hameed A, Sharif N, Qamar F, Abbas S. Health-related quality of life in patients with asthma, survey based study in Karachi. Journal of Bioequivalence \& Bioavailability. 2016; 8(4):179-84. [DOI:10.4172/jbb.1000290]

7. Urek MC, Tudorić N, Plavec D, Urek R, KoprivcMilenović T, Stojić M. Effect of educational programs on asthma control and quality of life in adult asthma patients. Patient Education and Counseling. 2005; 58(1):47-54. [DOI:10.1016/i.pec.2004.06.010] [PMID]

8. Gibson PG, Powell H, Wilson A, Abramson MJ, Haywood P, Bauman A, et al. Self-management education and regular practitioner review for adults with asthma. The Cochrane Database of Systematic Reviews. 2009;

(1):CD001117. [DOI:10.1002/14651858.CD001117]

9. Shames RS, Sharek P, Mayer M, Robinson TN, Hoyte EG, Gonzalez-Hensley F, et al. Effectiveness of a multicomponent self-management program in at-risk, school-aged children with asthma. Annals of Allergy, Asthma \& Immunology. 2004; 92(6):611-8. [DOI:10.1016/S1081-1206(10)61426-3]

10. Lee JE, Han HR, Song H, Kim J, Kim KB, Ryu JP, et al. Correlates of self-care behaviors for managing hypertension among Korean Americans: a questionnaire survey. International Journal of Nursing Studies. 2010; 47(4):411-7. [DOI:10.1016/j.ijnurstu.2009.09.011] [PMID]

11. Eftekhar H, Mirkamali SK, Tavafian SS, Mohammad K, Shahnazi H, Sharifirad G. Exploring self care in Tehran, Iran: A population based study. Journal of Research in Medical Sciences. 2012; 17(12):1144-9.
12. Mousing C, Lomborg K. Self care 3 months after attending chronic qbstrvctive pulmonary diease education. Patient Preference and Adherence. 2012; 6:1925. [DOI:10.2147/PPA.S28466] [PMID] [PMCID]

13. Rider SH, Dietrich MS, kidd N. Breast cancer treatmentrelated lymphedema self- care: Education, practice, symptons, and quality of life. Support Care Cancer. 2011; 19(5):631-7. [DOI:10.1007/s00520-010-0870-5] [PMID]

14. Godarzi M, Ahmadvand M, Farajollahi M. Study of Patient Teaching Importance from Point of Views in Azad University Nursing Students in Karaj in 1382. The Journal of Urmia Nursing and Midwifery Faculty. 2004; 2(2):68-77. [In Persian]

15. Goudarzi M, Ebrahimzadeh A, Rabie A, Saidipour B, Asghri Jafar Abadi M. The relationship between knowledge, attitude and self-efficacy in patients with type II diabetes in Karaj. Iranian Journal of Diabetes and Metabolism. 2011; 11(3):269-81. [In Persian]

16. Kadda O, Marvaki C, Panagiotakos D. The role of nursing education after a cardiac event. Health Science Journal. 2012; 6(4):634-46.

17. Schomerus G, Schwahn C, Holzinger A, Corrigan PW, Grabe HJ, Carta MG, et al. Evolution ofpublic-17 attitudes about mental illness: a systematic review and meta-analysis. Acta Psychiatrica Scandinavica. 2012; 125(6):440-52. 0447.2012.01826.x] [PMID]

[DOI:10.1111/j.1600-

18. Moore Z, Price P. Nurses' attitudes behaviours and towards pressure ulcer prevention. Journal of Clinical Nursing. 2004; 13(8):942-51. [DOI:10.1111/j.13652702.2004.00972.x] [PMID]

19. Wang WL, Herting JR, Tung YY. Adolescents' avoidance of secondhand smoke exposure: model testing. Western Journal of Nursing Research. 2008; 30(7):836-51. [DOI:10.1177/0193945908319251] [PMID]

20. Bourbonnais FF, Tousignant KF. The pain experience of patients on maintenance hemodialysis. Nephrology Nursing Journal. 2012; 39(1):13-9.

21. Zografos K, Marshak HH, Dyjack DT, Neis C. The effects of an adolescent asthma education intervention on knowledge, intention, behavior, self-efficacy and selfconsciousness. Californian Journal of Health Promotion. 2010; 8(1):60-71. [DOI:10.32398/cihp.v8i1.2031]

22. Kurtz ME, Kurtz J, Johnson SM, Beverly EE. Exposure to environmental tobacco smoke perceptions of African American children and adolescents. Preventive Medicine. 1996; 25(3):286-92. [DOI:10.1006/pmed.1996.0058] [PMID]

23. McCarthy MJ, Herbert R, Brimacombe M, Hansen J. Empowering parents through asthma education. Pediatric Nursing. 2002; 28(5):465-73.

24. Brito GM, Gois CF, Zanetti ML, Resende GG, Silva JR. Quality of life, knowledge and attitude after educational program for Diabetes. Acta Paul Enferm. 2016; 29(3):298-306. [DOI:10.1590/1982-0194201600042] 
25. Rajanandh M, Nageswari A, Ilango K. Impact of pharmacist provided education on konwledge, attitude, practice and quality of life in asthma patient in a south hospital. Journal of Medical Sciences. 2014; 14(5):25460. [DOI:10.3923/jms.2014.254.260]

26. Bidi F, Hassanpour K, Ranjbarzadeh A, Arab KA. Effectiveness of educational program on knowledge, attitude, self care and life style in patients with type II diabetes. 2013

27. Muchiri Jw, Gericke GJ, Rheeder P. Impact of nutrition education on diabetes knowledge and attitudes of adults with type 2 diabetes living in a resource-limited setting in South Africa: a randomised controlled trial. Journal of Endocrinology, Metabolism and Diabetes of South Africa. 2016; 21(2):26-34 [DOI:10.1080/16089677.2016.1200324]

28. Mazloomi S, Fallahzadeh $\mathrm{H}$. The relation of knowledge, attitude and self-management behaviors in asthmatic patients with controlling asthma. Zahedan Journal of Research in Medical Sciences. 2012; 14(1):49-55.

29. Zigheymat F, Ebadi A, Motahedian Tabrizi E, Alaf Javadi M, Hamedanizadeh F. Effect of training according to Health Belief Model (HBM) on health believing, knowledge and behavior of patients under CABG. Kowsar Medical Journal. 2009; 13(4):309-13.

30. Baba Beigi MA, Zibaeenzhad MJ, Aghasadeghi K, Jokar A, Shekar Foroush S, Khazraei H. The effecet of Education program on Hypertension management. International Cardio Vascular Research Journal. 2014; 8(3):94-8. [In Persian]

31. Goodarzi M, Javadi Bora MA, Farajollahi M. Impact of education via mobile phone on knowledge, attitude, practice and $\mathrm{HbA} 1 \mathrm{C}$ of patients with type 2 diabetes mellitus in Karaj-Iran. Interdisciplinary Journal of Virtual Learning in Medical Sciences. 2015; 6(3):8-19.

32. Baghiani Moghadam MH, Taheri G, Fallahzadeh H, Parsa M. The effect of instructional designed SMS based on Health Belief Model (HBM) on adoption of self-care behavior of patients with type II diabetes. Modern Care Journal. 2014; 11(1):10-8.

33. Abbaszadeha A, Borhanib F, Asadi N. Effects of Face-toface health-belief oriented education about risk factors on knowledge and attitude of myocardial infarction patients after discharge. Iranian Journal of Medical Education. 2012; 12(9):638-46.

34. Borji M, Azami M, Amirkhani M, Bastami E, Salimi K, Sayehmiri A, et al. The effect of education about the risk factors of myocardial infarction based on Health Belief Model on knowledge and attitudes of patients with myocardial infarction in Shahid Mustafa Hospital in Ilam. Nursing Journal of the Vunerable. 2016; 3(6):37-47. [In Persian] 